\title{
Tracheal damage after endotracheal intubation: comparison of two types of endotracheal tubes
}

\author{
D HONEYBOURNE, JC COSTELLO, C BARHAM
}

From the Chest and Anaesthetic Units, King's College Hospital, London SE5

ABSTRACT Twenty-eight patients who required endotracheal intubation for open-heart surgery were randomly allocated to one of two types of endotracheal tube. The tracheal mucosa was examined with a fibreoptic bronchoscope at the time of extubation, usually 24 hours after operation. The degree of oedema, inflammation, and ulceration was scored by the bronchoscopist, who also photographed the whole length of the trachea. An independent observer subsequently scored any tracheal damage from these photographs. Both observers found significantly less mucosal damage with the low-pressure, high-volume type of cuff than with the traditional highpressure, low-volume type. This difference may be related to the differences in lateral wall pressures exerted by the two types of cuff. The low-pressure type of cuff may be preferable in patients requiring prolonged endotracheal intubation.

Endotracheal and tracheostomy tubes are known to cause tracheal damage in a small proportion of patients. This damage can range from a temporary tracheitis of the mucosa underlying the cuff to permanent tracheal stenosis. One of the factors contributing to this damage is the pressure exerted by the cuff on the tracheal wall. Animal work $^{1}$ has shown that the degree of damage is related to the lateral pressure exerted by the endotracheal cuff on the adjacent mucosa. To prevent ischaemic damage the cuff should exert a lateral wall pressure no greater than the capillary perfusion pressure of the mucosa, the mean capillary perfusion in the human tracheal mucosa being about $20 \mathrm{~mm} \mathrm{Hg}(2 \cdot 7 \mathrm{kPa})$.

The aim of the study was to compare the effects of two types of endotracheal tube, a high-pressure, low-volume type (Portex Blueline, PBL), which is in common use in operating theatres in this country, and a low-pressure, high-volume type (Extracorporeal Lanz, EL), which is not widely used at present. The fundamental difference between the two tubes is that the EL tube incorporates a valve mechanism in the outer pilot balloon. This external pressure-regulating valve controls the pressure exerted by the cuff on the wall of the trachea. Once the cuff is inflated the valve maintains a maximum pressure of $20-25 \mathrm{~mm} \mathrm{Hg}(2 \cdot 7-3.3 \mathrm{kPa})$ on the tracheal wall during expiration, any excess air in the

Address for reprint requests: Dr D Honeybourne, Department of Thoracic Medicine, Hope Hospital, Salford M6 8HD. cuff escaping via the valve into the external pilot balloon. The PBL tube contains no such device.

In vitro work ${ }^{2}$ using an adult model trachea to investigate a variety of endotracheal tubes showed that the sealing point of the cuff in the model was achieved at a lower pressure in the EL tube than in the PBL and similar tubes with no valve mechanism. The lower intracuff pressure at the sealing point led to a lower lateral wall pressure on the model trachea. If $10 \mathrm{ml}$ more air than was required to seal the cuff in the trachea was blown into the cuff, the intracuff pressure rose in the PBL tube, leading to an increase in the lateral wall pressure. A.s a result of the valve mechanism, however, a further $10 \mathrm{ml}$ of air blown into the EL tube produced no such rise in the intracuff pressure; thus the lateral wall pressure remained at or below the theoretical mean capillary perfusion pressure on the underlying tracheal wall. This in vitro study suggests therefore that the damage to the tracheal wall may be less with the lowpressure, high-volume type of tube (EL). Studies on cadaver tracheas have yielded similar conclusions, ${ }^{3}$ and we decided to carry out a comparative study of the two types of tube on patients undergoing openheart surgery.

\section{Method}

The study was performed on 28 patients presenting for open-heart surgery who were to have elective postoperative ventilation for about 24 hours. All patients gave informed consent and were then ran- 
domly assigned to recive a PBL or an EL type of endotracheal tube. All patients had a nasogastric tube inserted and were then intubated. After intubation the anaesthetist inflated the cuff until the sealing pressure had been reached-this was dectectable by the lack of any leak around the tube on inflation of the lungs. Fibreoptic bronchoscopy was then performed during extubation to assess tracheal damage.

When ready for extubation the patient was ventilated with $100 \%$ oxygen for two minutes and after he had had a careful explanation of the procedure the back of the pharynx was sucked out and the fibreoptic bronchoscope (Olympus B3) with camera attached (Olympus camera S/C 16-2) was inserted into the endotracheal tube and passed down as far as the main carina. The cuff was then deflated and the endotracheal tube fed back up and over the bronchoscope by an assistant. The bronchoscope was then slowly withdrawn and the tracheal mucosa was inspected from the main carina to the subglottic region and from six to nine photographs were taken.

The bronchoscopist scored separately any oedema, inflammation, or ulceration seen, each on the scale $0-3$, so that the range of points was zero for no damage whatever to 9 where oedema, inflammation, and ulceration were all severe. The bronchoscopist was aware of which endotracheal tube had been used on a particular patient because of the different external appearance of the pilot balloons. The photographs taken at extubation were, however, later analysed by an independent observer, also an experienced bronchoscopist, who was unaware of which tube had been used in any patient. This observer used an identical scoring system to the bronchoscopist. Results were analysed by Student's $t$ test and Wilcoxon's rank sum test.

\section{Results}

The mean tube size and duration of intubation were not significantly different between the two groups (table 1). The mean age of the EL group, however, was significantly higher than that of the PBL. There were two women in each group.

The linear correlation between the scores of the bronchoscopist (A) and the independent observer scoring from the photographs (B) was $r=0.82$, and according to the both scores the PBL tube caused
Table 2 Scores of the bronchoscopist $(A)$ and the independent observer scoring from photographs $(B)$ for the damage caused by the two types of endotracheal tube

\begin{tabular}{lll}
\hline Tube & Mean score A & Mean score $B$ \\
\hline Portex Blue Line & 5.9 & 5.8 \\
Lanz & 2.7 & 3.5 \\
p value & $<0.01$ & $<0.05$ \\
\hline
\end{tabular}

significantly more damage than did the EL tube (table 2).

\section{Discussion}

This in vivo study has shown that there is significantly less damage to the tracheal mucosa after about 24 hours of intubation with the lowpressure, high-volume type of endotracheal tube (EL) than the traditional high-pressure, low-volume type (PBL). This tends to confirm the in vitro finding ${ }^{2}$ that the lateral wall pressure at the sealing point differed between the two types of tube. Thus the observed changes on the tracheal wall may be related to the differences in the lateral wall pressure exerted by their cuffs.

Other factors known to contribute to tracheal damage include the size of the endotracheal tube in relation to the tracheal lumen, the cardiovascular state of the patient during intubation, the duration of intubation, the material from which the cuff is manufactured, and the age of the patient. With the exception of age all these variables were eliminated by allocating our patients randomly to one of the two groups. If anything, the slightly greater mean age of the EL group would have masked an even bigger difference between the two groups, as it is known that tracheal damage is more likely in children or older adult patients. ${ }^{4}$ None of our patients suffered any long-term clinical sequelae resulting from intubation with either tube. While permanent tracheal damage is an infrequent complication of endotracheal intubation it is nevertheless a hazard that may be lessened in the light of this study.

Theoretically aspiration of gastric or pharyngeal secretions may seem more likely with a low-pressure type of cuff, but his has not occurred in our experience and should not happen if the cuff is initially

Table 1 Details of patients allocated to each type of endotracheal tube

\begin{tabular}{lllll}
\hline & No of patients & Mean age $\pm S D(y)$ & Mean tube size (mm) & $\begin{array}{c}\text { Mean duration of } \\
\text { intubation }(\mathrm{h}) \pm S D\end{array}$ \\
\hline Portex Blue Line (PBL) & 13 & $* 48.5 \pm 11 \cdot 7$ & $9 \cdot 0$ & $24.4 \pm 7.7$ \\
Extracorporeal Lanz (EL) & 15 & $* 56.6 \pm 10.6$ & $9 \cdot 0$ & $22.9 \pm 2.1$ \\
\hline
\end{tabular}

${ }^{*} \mathrm{p}<0.05$ (Student's $t$ test). 
inflated to achieve the sealing point. The valve mechanism increases the cost of this type of endotracheal tube and therefore its routine use for all patients requiring intubation cannot be recommended. Conceivably, however, the difference in the damage inflicted by the two tubes might be greater with prolonged intubation than after about 24 hours, and a small proportion of the more severely affected patients could develop permanent tracheal damage. In those patients who can be expected to need prolonged intubation and mechanical ventilation (for example, for adult respiratory distress syndrome) a low-pressure, high-volume type of cuff should be preferred to the traditional high-pressure, low-volume type.

Mucosal biopsy specimens taken during the procedure could theoretically add more information but we think that biopsy is probably unjustified by virtue of being a highly invasive technique and also not a very practical method, in view of the short time available to view the trachea during extubation. Our technique of assessing tracheal damage via endotracheal tubes should be useful in further studiesfor instance, using different types of high-volume, low-pressure tubes.

We thank Professor L Strunin for this help and advice with this study.

\section{References}

1 Nordin U. The trachea and cuff-induced tracheal injury. Acta Otolaryngol 1977;345, suppl:1-71.

${ }^{2}$ Leigh JM, Maynard JP. Pressure on the tracheal mucosa from cuffed tubes. $\mathrm{Br} M e d \mathrm{~J} 1979 ; \mathrm{i}: 1173-4$.

${ }^{3}$ Crawley BE, Cross DE. Tracheal cuffs. Anesthesia 1975;30:4-11.

${ }^{4}$ Mathias DB, Wedley JR. The effects of cuffed endotracheal tubes on the tracheal wall. Br J Anaesth $1974 ; 46: 849-52$. 